\section{Revista Mexicana de Astronomia y Astrofisica}

Revista Mexicana de Astronomía y Astrofísica ISSN: 0185-1101

rmaa@astroscu.unam.mx

Instituto de Astronomía

México

Steffen, W.; Tamayo, F.; Koning, N.

CRISS-CROSS MAPPING BD+30 3639: A NEW KINEMATIC ANALYSIS TECHNIQUE

Revista Mexicana de Astronomía y Astrofísica, vol. 40, 2011, p. 202

Instituto de Astronomía

Distrito Federal, México

Available in: http://www.redalyc.org/articulo.oa?id=57121297109

How to cite

Complete issue

- More information about this article

Journal's homepage in redalyc.org

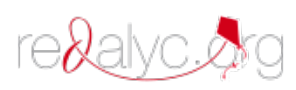

Scientific Information System Network of Scientific Journals from Latin America, the Caribbean, Spain and Portugal Non-profit academic project, developed under the open access initiative 
RevMexAA (Serie de Conferencias), 40, 202-202 (2011)

\title{
CRISS-CROSS MAPPING BD+30 3639: A NEW KINEMATIC ANALYSIS TECHNIQUE
}

\author{
W. Steffen, ${ }^{1}$ F. Tamayo, ${ }^{1}$ and N. Koning ${ }^{2}$
}

We introduce a new method to analyze proper motion data. The method is called "crisscross" mapping. It emphasizes regions where proper motion vector extensions cross or converge. From a superposition of lines through the vectors, a map is generated which helps to interpret the kinematic data. The new mapping technique is applied to the young planetary nebula $\mathrm{BD}+30$ 3639. The data are approx. 200 internal proper motion measurements from Li, Harrington, \& Borkowski (2002). From the criss-cross mapping of $\mathrm{BD}+30$ 3639, we conclude that the kinematic center is approximately 0.5 arcsec offset to the South-East from the central star. The mapping also shows evidence for a nonhomologous expansion of the nebula that is consistent with a disturbance aligned with the bipolar molecular bullets.

We define the criss-cross mapping by the following procedure: replace proper motion vectors with a thin line that extends over the complete area covered by the nebula. Assign a finite constant brightness to every line. Generate an image by adding together all lines. The result is convolved with a suitable kernel like a gaussian with a width that is larger than the average separation between the vectors. In the regions where vectors converge the image will increase in brightness and reveal where most velocity vectors meet.

Our numerical scheme determines whether an image pixel $(i, j)$ is on a line going through the vector indexed $k$. If so, then the weight value $w_{k}$ is added to the pixel

$$
I_{\mathrm{d} i, j}=\sum_{k=1}^{\mathrm{n}_{k}} w_{k} \delta\left(d_{i, j ; k, s}\right)
$$

where $i, j$ are the indices of the image pixels, $k$ is the velocity vector index and the total number of velocity vectors is $\mathrm{n}_{k}$. The distance of the image pixel $i, j$ to the vector line $k$ is $\mathrm{d} i, j$. In equation (1)

\footnotetext{
${ }^{1}$ Instituto de Astronomía, Universidad Nacional Autónoma de México, Km 103 Carr. Tijuana-Ensenada, CP 22860, Ensenada, B.C., Mexico (wsteffen@astrosen.unam.mx).

${ }^{2}$ University of Calgary, Calgary, Canada.
}

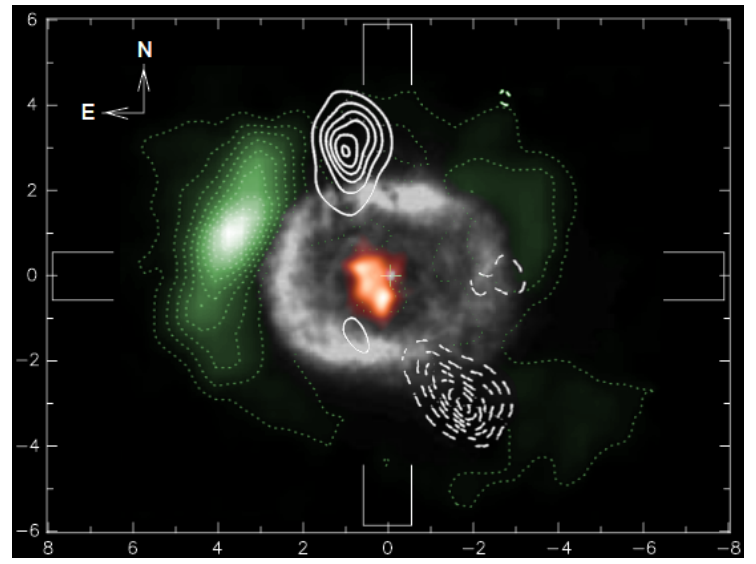

Fig. 1. Several types of maps have been combined: optical (grey, from LHB2002), $\mathrm{H}_{2}$ (contoured green, from Shupe et al. 1998), CO (white contour, from Bachiller et al. 2000) and the criss-cross map (red, this work).

$\delta(d)$ is a top-hat function with a value of 0 or 1 . The total width of the top-hat function -which represents the line width- is $s$.

We have implemented the procedure in the morpho-kinematic 3D modeling software Shape (Steffen et al. 2011, freely available from http: //www.astrosen.unam.mx/shape). It allows one to generate criss-cross maps from observations and models.

This work has been supported by grants from CONACYT 49447 and UNAM PAPIIT IN100410. N.K. received additional support from the Natural Sciences and Engineering Council of Canada (NSERC) and from the Killam Trusts.

\section{REFERENCES}

Bachiller, R., Forveille, T., Huggins, P. J., Cox, P., \& Maillard, J. P. 2000, A\&A, 363, 25

Li, J., Harrington, J. P., \& Borkowski, K. 2002, ApJ, 123, 2676

Shupe, D. L., et al. 1998, ApJ, 498, 267

Steffen, W., Koning, N., Wenger, S., Morisset, C., Magnor, M., 2011, IEEE Trans. Visualization Computer Graphics, in press, arXiv:1003.2012 